\title{
Acute kidney injury with cardiorenal syndrome; experience from a tertiary care renal unit in Pakistan
}

\author{
Rubina Naqvi*i \\ Department of Nephrology, Sindh Institute of Urology and Transplantation (SIUT), Karachi, Pakistan
}

\section{A R T I C L E IN F O}

\section{Article Type:}

Original

\section{Article History:}

Received: 10 May 2020

Accepted: 19 October 2020

Published online: 10 November 2020

\section{Keywords:}

Acute kidney injury (AKI), Kidney Disease Improving Global Outcome (KDIGO) guidelines, Cardio-renal syndrome, Acute myocardial infarction, Congestive cardiac failure, Infective endocarditis

\begin{abstract}
A B S T R A C T
Introduction: Acute kidney injury (AKI) is a commonly recognized clinical problem after many morbid conditions related to heart like congenital heart disease surgery, acute or chronic congestive heart failure, acute myocardial infarction, infective endocarditis or cardiomyopathies. Cardio-renal syndrome (CRS) includes a spectrum of disorders involving both the heart and kidneys simultaneously; here acute or chronic dysfunction in one organ may induce acute or chronic dysfunction in the other.

Objectives: To report here, case series of patients with AKI developing in association with CRS. We aim to report different causes of CRS and outcome of patients in this group of patients.

Patients and Methods: Subjects for the study reported here comprised a cohort of 34 patients coming to this institution with AKI in association of CRS. AKI was defined according to KDIGO guidelines and CRS based on consensus conference of ADQI in 2012. Type lor type2 CRS are included in the study. All patients had normal size kidneys on ultrasonography.

Results: Thirty-four patients with AKI and CRS were brought to this institute from January 1990 to December 2014; this was contributing 1\% to medical causes of total AKI. Among these 25 were males and 9 females; mean age of these patients was $54.06 \pm 14.106$ years. Causes of CRS were acute myocardial infarction (ST elevated), congestive cardiac failure, infective endocarditis and dilated cardiomyopathy. More than two third of patients were either oliguric or anuric on presentation. Fluid replacement and/or inotropic support required in $79 \%$. Renal replacement therapy in form of hemodialysis was conducted in $64.7 \%$ and intermittent peritoneal dialysis in one patient. Complete renal recovery was observed in 19 (56\%) patients, while $12(35 \%)$ died during acute phase of illness. CKD-V developed in one patient, 2 patients lost long term follow up, but became dialysis free and renal functions were in improving trends, they were labeled as partial recovery. Secondary insults like hypotension, aggressive diuresis, and volume loss from gastro-intestinal tract or infection were evaluated for any corelation with outcome but statistically no significant difference was found.

Conclusion: CRS can be severe life-threatening condition especially when patients present with circulatory collapse. Diuretics must be used cautiously in patients with congestive cardiac failure. Infective endocarditis with acute right heart failure can lead to CRS.
\end{abstract}

Implication for health policy/practice/research/medical education:

The cardio-renal syndrome includes a spectrum of disorders involving both heart and kidneys simultaneously; here acute or chronic dysfunction in one organ may induce acute or chronic dysfunction in the other.

Please cite this paper as: Naqvi R. Acute kidney injury with cardiorenal syndrome; experience from a tertiary care renal unit in Pakistan. J Renal Inj Prev. 2021; 10(4): e29. doi: 10.34172/jrip.2021.29.

\section{Introduction}

The term cardio-renal syndrome (CRS) includes a spectrum of disorders involving both the heart and kidneys in which acute or chronic dysfunction in one organ may induce acute or chronic dysfunction in the other. There have been described five types of CRS based on consensus conference of ADQI in 2012. Type 1 CRS or acute CRS is the category where acute heart failure (AHF) causes acute kidney injury (AKI), for example; acute coronary syndrome (ACS) resulting in cardiogenic shock and AKI due to hemodynamic instability. Type 2 is a chronic cardiac failure (CCF) resulting in AKI or chronic kidney 
disease (CKD); for example sudden and rapid diuresis or any other insult in established CCF may result in AKI. Type 3 CRS is AKI resulting in AHF and type 4 CRS is characterized by CKD causing AHF or CCF, whereas type 5 CRS is where acute or chronic systemic disorders lead to cardiac and renal dysfunction simultaneously, examples are amyloidosis, sepsis and cirrhosis (1).

An interesting fact described is a significantly higher proportion of AHF patients developing type 1 CRS compared to ACS or major cardiac surgery (2). While type 1 CRS is characterized by acute and rapid worsening of the heart leading to AKI. Other compounding effects of type 1 CRS include neurohormonal activation, hypothalamicpituitary stress reaction, inflammation and immune cell signaling, oxidative stress and failure of counter-regulatory mechanisms (3).

The simplistic view of CRS is that a relatively normal kidney is dysfunctional because of a diseased heart, with the assumption that, in the presence of a healthy heart, the same kidney would perform normally. The CRS includes a variety of acute or chronic conditions, where the primary failing organ can be either the heart or the kidney. AHF may be divided into four subtypes; hypertensive pulmonary edema with preserved left ventricular (LV) systolic function, acutely decompensated CCF, cardiogenic shock, and pre-dominant right ventricular failure (4).

The standardized criteria for the diagnosis of AKI greatly improved after KDIGO guidelines for definition of AKI with emphasis on small fluctuations in serum creatinine and urine output; however, they may not represent true renal tubular injury when observed in the context of diuresis in the setting of AHF (5).

\section{Objectives}

The current study is aimed to report CRS from tertiary care renal unit where these patients were referred from cardiology or internal medicine facilities after being found derangement in renal functions and/or decline in urine output.

\section{Patients and Methods \\ Study design}

In this retrospective study clinical records of all adult patients ( $\geq 18$ years) were reviewed who were admitted to Sindh institute of urology and transplantation (SIUT) from January 1990 - December 2014 and in whom the diagnosis of AKI and CRS was established. AKI was defined according to KDIGO (Kidney Disease Improving Global Outcomes) guidelines (6) in retrospect while CRS was defined as rapid worsening of cardiac function, leading to AKI (type land type 2 CRS are included).

The variables recorded from each patient record from day of admission include age, gender, history, duration of insult, oliguria, anuria, hemoglobin, total leukocyte count, platelet count, prothrombin time, activated partial thromboplastin time, peripheral blood film (which include reporting of presence or absence of malarial parasite), blood urea, serum creatinine, serum sodium, serum potassium, venous bicarbonate, serum $\mathrm{LDH}$, creatine phosphokinase, troponin I, alanine aminotransferase (ALT), aspartate aminotransferase (AST), urine dipstick, urine microscopy, blood and urine microbial cultures in selected cases, ultrasonography, use of parenteral fluids before renal replacement therapy, renal replacement therapy, sessions of hemodialysis or days on peritoneal dialysis and outcome.

Patients were labelled as completely recovered when regained normal renal functions and sustained it in long term follow up. Partially recovered when became dialysis free but not turned to baseline and lost follow up before 90 days. CKD was labeled for patients who did not recovered normal renal functions beyond 90 days.

Patients with lack of baseline serum creatinine or diabetic patients were not included in this study.

\section{Data analysis}

Statistical analysis was done on SPSS version 20.0. Mean \pm standard deviation was expressed or median with inter-quartile range provided for continuous variables. Frequencies and percentages were computed for categorical variables. Chi-square test was used to determine the proportional difference between outcomes with different presenting symptoms. $P$ value $<0.05$ was considered as significant.

\section{Results}

During the study period of 25 years, 34 patients fulfilled the criteria and were included in the current study. Among these 25 were males and 9 were female other demographics and laboratory values on day of admission to this institution are given in Table 1. Primary insult is given in Figure 1. Combination of more than one or secondary insults is given in Table 2. Four (11.8\%) patients were anuric and $22(64.7 \%)$ oliguric on presentation, while rest had non-oliguric AKI. Urinalysis was available for 26 patients, on dipstick proteinuria from trace to $2+$ was found and microscopic hematuria was reported in 15 of them. Echocardiography findings available for 21 other patients were unstable to be moved to department for procedure, 18 of these had preserved LV function and ejection fraction (EF) more than 45 , while 3 revealed suppressed ventricular function. Patients with infective endocarditis (IE) who were all IV drug abusers shown vegetations of variable sizes on tricuspid valve.

Half of patients (17 cases) required inotropic support on arrival, hydration was done in 27 (79.4\%), renal replacement in form of hemodialysis required in 22 (64.7\%) and intermittent peritoneal dialysis during the initial part of study was done in one patient, this patient was recovered after 2 days of this therapy. 
Table 1. Baseline demographics and laboratory values

\begin{tabular}{|c|c|c|c|}
\hline Parameters & Mean \pm SD & Median & Interquartile range \\
\hline Age (years) & $54.06 \pm 14.106$ & 57.50 & $44.25-64.25$ \\
\hline Duration of insult (days) & $7.88 \pm 5.871$ & 6 & $4-9.25$ \\
\hline Hemoglobin (g/dL) & $11.385 \pm 2.1225$ & 11.250 & $10.20-13$ \\
\hline Total leukocyte count & $14.906 \pm 6.085$ & 13.30 & $10.725-19.225$ \\
\hline Platelet $\times 10^{3} / \mu \mathrm{L}$ & $226.26 \pm 129.838$ & 222 & $120.50-301.00$ \\
\hline Urea (mg/dl) & $255.79 \pm 113.193$ & 226 & $179.50-368.50$ \\
\hline Creatinine (mg/dL) & $8.512 \pm 5.356$ & 7.350 & $5.525-9.150$ \\
\hline $\mathrm{LDH}(\mathrm{U} / \mathrm{L})$ & $1753.24 \pm 2468.417$ & 786 & $471.00-1370.00$ \\
\hline Creatine phosphokinase (U/L) & $716.06 \pm 679.847$ & 460 & $156-1106$ \\
\hline Sodium (mEq/L) & $131.79 \pm 6.163$ & 132 & $128.50-135.50$ \\
\hline Potassium (mEq/L) & $5.174 \pm 1.3263$ & 5.2 & $4.100-6.12$ \\
\hline AST (U/L) & $381.42 \pm 1211.809$ & 59 & $30-108$ \\
\hline $\operatorname{ALT}(U / L)$ & $72.61 \pm 104.196$ & 48 & $23-81$ \\
\hline
\end{tabular}

Table 2. Combinations of secondary insults

\begin{tabular}{ll}
\hline Primary insult & Other combination/secondary insult \\
\hline Acute myocardial infarction $(n=18)$ & $\begin{array}{l}\text { Hypotension requiring inotropes }(n=7), \text { Hypotension + mechanical ventilation ( } n=1), \\
\text { hypotension + pulmonary edema }(n=1)\end{array}$ \\
Congestive cardiac failure $(n=8)$ & Diuretics $(n=5)$, multiple vomitings $(n=1), U T I(n=1)$, pulmonary edema $(n=1)$ \\
Infective endocarditis $(n=7)$, all IV drug abusers & Febrile illness $(n=6)$, Diarrhea $(n=1)$ \\
Dilated cardiomyopathy $(n=1)$ & Multiple vomitings $(n=1)$ \\
\hline
\end{tabular}

A total of 19 (56\%) recovered normal renal functions, 12 (35\%) died during acute phase of illness, 5 of these 12 died within 48 hours of reaching to this facility. One patient developed CKD-VD, while 2 who were discharged from hospital with improving trends of renal functions lost follow up thus labelled as partially recovered.

Outcome was assessed with combinations of primary and secondary insults and recovery or death in these terms was analyzed. CCF with different combinations revealed $P$ value of 0.898 and acute myocardial infarction (MI) with hypotension on presentation revealed $P$ value of 0.067 (chi-square test).

\section{Discussion}

During this study period of 25 years total of 5,623 patients with AKI were seen at this institution $60 \%$ of these were due to medical causes and $1 \%$ of medical causes was CRS. A study from the US has reported more than 1 million hospital admissions with AHF or de-compensated CCF and development of AKI has been reported in 70\% of patients who were in cardiogenic shock; this study has reported AKI more severe in patients with impaired LV function and low EF. Furthermore, impaired renal function is consistently found as an independent risk factor for 1-year mortality in AHF patients, including patients with ST-segment elevation MI (4). From our country data from cardiology units or internal medicine in regards to how many patients developing AKI is not available. A recently published study conducted on surgical patients undergoing congenital heart disease surgery on cardiac bypass; revealed $28 \%$ of patients developing AKI, both children and adult population were included in this study (7). When search made for other regional studies; a case report found from India reporting diuretic resistance and later developing CRS was found (8). Another original

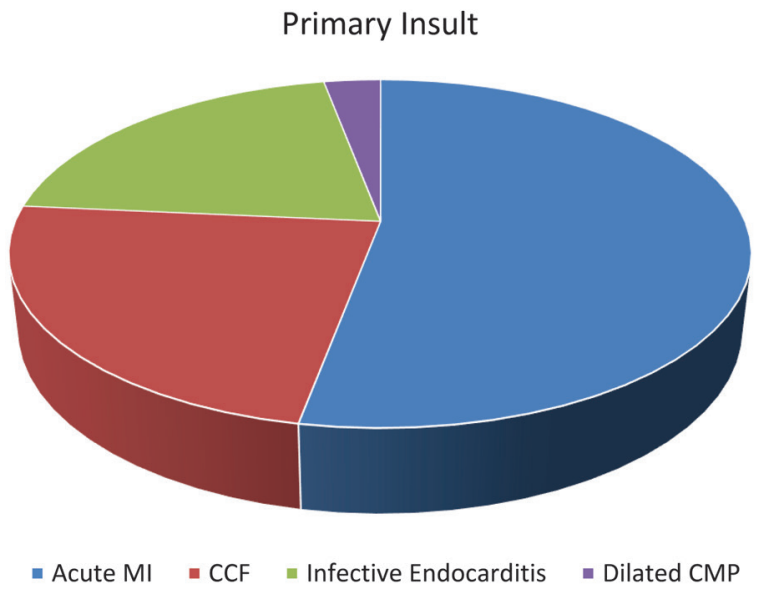

Figure 1. Primary insult. 
article from India highlighting predictors of CRS type 1 in patients with acute right ventricular dysfunction indicated diabetes and presentation in state of shock as poor prognostic markers in their series (9).

Our studied population included 18 cases of acute MI (ST elevation) all of them were hypotensive (many requiring inotropes), these hemodynamic derangements can cause decreased renal perfusion and ultimately decline in glomerular filtration rate (GFR). Other possible influence for development of CRS is use of reninangiotensin-aldosterone system inhibitors which can halt autoregulatory response to reduced perfusion pressure and renal blood flow leading to decreased GFR (10). This can be reflected by one or both criteria; like rise in serum creatinine from baseline value or decline in urine output. KDIGO as well as other definitions of AKI include both. In the current study, $76.5 \%$ of patients were either oliguric or anuric on presentation and all had raised serum creatinine.

Other category of our patients who had established diagnosis of CCF, they usually have diuretics on their prescription. However, hypothetically sometimes decrease diuretic response may result from diminished effectiveness secondary to post diuretic sodium retention (4). Sometimes patients may discontinue taking medications and when turning to physicians they administer larger doses of diuretic to get a quicker response; this can be an iatrogenic mechanism of aggravating AKI.

In our study among 8 of our CCF patients 5 had received high doses of diuretics recently, one had volume loss via excessive vomiting, one had concomitant urinary tract infection and one arrived with pulmonary edema. Assessment of patients with CCF and their treatment for resolution of problem has remained a topic of debate for long time. Especially when congestion is only 'tip of ice-berg' and actual status of cardiac dysfunction leading to increased filling pressure and towards pulmonary circulation and central venous system is unknown. Some studies have assessed acute tubular injury by doing urinary markers and found no direct correlation of acute tubular injury with aggressive diuresis (11).

Another famous trial the ROSE-AHF trial was unique in its use of very high diuretic doses to cause brisk decongestion, in this trial as per protocol, all patients received 2.5 times their home dose of loop diuretics as background therapy, whereas half of patients were getting low-dose nesiritide and other half low dose dopamine in both arms. This resulted in $8.4 \mathrm{~L}$ urine during the 72 hour study period in the placebo arm and required rapid decongestion is achieved over here (11-13).

Endocarditis associated with right heart failure in IV drug abusers was the case in 7 of our patients, their presentation in 6 of 7 was with high grade fever, positive blood cultures and finding of vegetations of variable sizes on tricuspid valve, while one had volume loss via gastrointestinal tract in form of diarrhea this patient also found to have positive findings on echocardiography. Renal biopsy was done in two patients and findings were mild tubular injury with no glomerular involvement was found in both of them. AKI with infective endocarditis has previously been reported in literature and cardiac surgery for infective endocarditis or heart failure both has been recognized as high risk factors for development of AKI (14).

The echocardiographic findings in the current study may be biased as it was performed when patients were stable enough to be moved to department. Moreover, echocardiography carried out during stay in intensive care or high dependency are not very reliable to document findings as proper probe and machine was not used to assess all parameters and was performed by nephrologists not the cardiologists.

\section{Conclusion}

CRS can be severe life threatening condition especially when a patient presents with circulatory collapse. Diuretics must be used cautiously in patients with congestive cardiac failure. Infective endocarditis with acute right heart failure can lead to CRS. In both chronic and acute situations, an appreciation of the interaction between heart and kidney during dysfunction of each or both organs has practical clinical implications. The depth of knowledge and complexity of care necessary to offer best therapy to these patients demands a multidisciplinary approach, combining the expertise of cardiology, nephrology, and critical care.

\section{Limitations of the study}

The study was done in retrospect looking at records of patients who were brought to a tertiary renal care unit. Therefore, prevalence of AKI or CRS in population can not be commented. A study at multidisciplinary hospital can better highlight the prevalence of the condition.

\section{Acknowledgements}

Valuable help of Mr. Iqbal Mujtaba (Research Dept. SIUT) for statistical analysis of data is gratefully acknowledged.

\section{Author's contribution}

$\mathrm{RN}$ is the single author of the manuscript.

\section{Conflicts of interest}

The author declares that she has no competing interests.

\section{Ethical issues}

The study protocol was in accordance with the Declaration of Helsinki and SIUT's Institutional Ethical Review Committee has granted permission for publishing this data (approval number\# SIUT- ERC-2020/A-216). Additionally, ethical issues (including plagiarism, data 
fabrication, double publication) have been completely observed by the author.

\section{Funding/Support}

None.

\section{References}

1. House AA, Anand I, Bellemo R, Cruz D, Bobek I, Anker SD, et Definition and classification of Cardio-Renal Syndromes: workgroup statements from the 7th ADQI Consensus Conference. Nephrol Dial Transplant. 2010;25:1416-20. doi: $10.1093 /$ ndt/gfq136.

2. Vandenberghe W, Gevaert S, Kellum JA. Acute kidney injury in cardiorenal syndrome type 1 patients: a systematic review and meta-analysis. Cardiorenal Med. 2016;6:116-28.

3. Ronco C, Cicoira M, McCullough PA. Cardiorenal syndrome type 1: pathophysiological crosstalk leading to combined heart and kidney dysfunction in the setting of acutely decompensated heart failure. J Am Coll Cardiol. 2012; 60: 1031-42.

4. Ronco C, Haapio M, House AA, Anavekar N, Bellomo R. Cardiorenal syndrome. J Am Coll Cardiol. 2008;52:152739. doi: 10.1016/j. jacc.2008.07.051.

5. Rangaswami J, Bhalla V, Blair JEA, Chang TI, Costa S, Lentine KL, et al. Cardiorenal syndrome: classification, pathophysiology, diagnosis and treatment strategies. Circulation. 2019;139:e840-78.

6. KDIGO: Definition and classification of AKI. KI Suppl. 2012;2:19-36

7. Ali F, Khan M, Mirza B, Qureshi S, Abbas Q. Acute kidney injury after congenital heart disease surgery: a single-center experience in a low- to middle-income country. Cureus. 2020;12(4):e7727. doi: 10.7759/cureus.7727.

8. Aundhakar SC, Mahajan SK, Mane MB, Lakhotia AN, Mahajani VV. Cardiorenal syndrome: resistant to diuretics and sensitive to ultrafilteration. J Cardiovasc Dis Res. 2012;3:173-5.

9. Tandon R, Mohan B, Chhabra ST, Aslam N, Wander GS Clinical and echocardiographic predictors of cardiorenal syndrome type1 in patients with acute ischemic right ventricular dysfunction. Cardiorenal Med. 2013;3:239-45.

10. Damman K, Tang WH, Testani JM, McMurray JJ. Terminology and definition of changes renal function in heart failure. Eur Heart J. 2014;35:3413-6. doi: 10.1093/ eurheartj/ehu320.

11. Ahmad T, Jackson K, Rao VS, Tang WH, Brisco-Bacik MA, $\mathrm{Chen} \mathrm{HH}$, et al. Worsening renal function in patients with acute heart failure undergoing aggressive diuresis is not associated with tubular injury. Circulation. 2018;137:201628. doi: 10.1161/CIRCULATIONAHA.117.030112.

12. Felker GM, Lee KL, Bull DA, Redfield MM, Stevenson LW, Goldsmith SR, et al. NHLBI Heart Failure Clinical Research Network. Diuretic strategies in patients with acute decompensated heart failure. N Engl J Med. 2011;364:797805. doi: 10.1056/NEJMoa1005419.

13. Hanberg JS, Tang WHW, Wilson FP, Coca SG, Ahmad T, Brisco MA, et al. An exploratory analysis of the competing effects of aggressive decongestion and highdose loop diuretic therapy in the DOSE trial. Int J Cardiol. 2017;241:277-82. doi: 10.1016/j.ijcard.2017.03.114.

14. Gagneux-Brunon A, Pouvaret A, Maillard N, Berthelot P, Lutz MF, Cazorla C, et al. Acute kidney injury in infective endocarditis: a retrospective analysis. Med Mal Infec2019;49:527-33. doi: 10.1016/j.medmal.2019.03.015.

Copyright (c) 2021 The Author(s); Published by Nickan Research Institute. This is an open-access article distributed under the terms of the Creative Commons Attribution License (http://creativecommons.org/licenses/by/4.0), which permits unrestricted use, distribution, and reproduction in any medium, provided the original work is properly cited. 